\title{
Subpixel Accuracy Analysis of Phase Correlation Shift Measurement Methods Applied to Satellite Imagery
}

\author{
S.A. Mohamed, A.K. Helmi \\ Division of Data Reception Analysis and Receiving \\ Station Affairs, National Authority for Remote Sensing \\ and Space Sciences, Cairo, Egypt
}

\author{
M.A. Fkirin, S.M. Badwai \\ Department of Industrial Electronic Engineering and \\ Control, Faculty of Electronic Engineering, \\ MenoufiaUniversity, Menoufia, Egypt
}

\begin{abstract}
-the key point of super resolution process is the accurate measuring of sub-pixel shift. Any tiny error in measuring such shift leads to an incorrect image focusing. In this paper, methodology of measuring sub-pixel shift using Phase correlation (PC) are evaluated using different window functions, then modified version of (PC) method using high pass filter (HPF) is introduced . Comprehensive analysis and assessment of (PC) methods shows that different natural features yield different shift measurements. It is concluded that there is no universal window function for measuring shift; it mainly depends on the features in the satellite images. Even the question of which window is optimal of particular feature is generally remains open. This paper presents the design of a method for obtaining high accuracy sub pixel shift phase correlation using (HPF).The proposed method makes the change in the different locations that lack of edges easy.
\end{abstract}

Keywords-phase correlation (PC); high pass filter (HPF); window function; sub-pixel shift.

\section{INTRODUCTION}

Sub pixel image shift estimation is a fundamental task for high performance image processing techniques such as image fusion and super resolution, which have been extensively used for applications in remote sensing, medical imaging, surveillance and computer vision. In [1], frequency based shift calculated methods using phase correlation (PC) have been widely used because of its accuracy and low complexity for shift motion due to translation, rotation or scale changes between images. The PC method for images alignment relies mainly on the shift property of the Fourier transform to estimate the translation between two images [2]. It is extended to estimate rotation and scale changes by using log-polar coordinate changes [3]. Originally limited to discover only integer pixel translations, the algorithm can be naturally extended to provide sub pixel accuracy [4], but at a higher computational cost. On the other hand, newer approaches have also allowed obtaining sub pixel accuracy with much less complexity [5], and some of its latest variations have reported enhanced accuracy performances $[6,7,8]$.

Using phase correlation with window; the window function plays a crucial role in determining the phase (wave front) because it significantly influences phase error. Window functions are used in harmonic analysis to reduce the undesirable effects related to the spectral leakage. They impact on many attributes of a harmonic processor which include detestability, resolution, dynamic range, confidence and ease of implementation [9]. Several standard windows are also used to optimize the requirements of a particular application in signal processing. Window functions have been successfully used in various areas of signal processing and communications such as, spectrum estimation, speech processing, digital filter design, and in other related fields such as, beam forming. A complete review of many window functions and their properties was presented by Harris [10].

All window functions are designed to reduce the side lobes of the spectral output of Fast Fourier transform (FFT) routines. Whilst applying the window function reduces the side lobe leakage, it causes the main lobe to broaden thus, reducing the resolution. This is a trade-off that has to be made, one should choose the window function, which best suits the application. Some windows which have small side-lobe level and quick fall off rate have been used to reduce the measurement error of dielectric loss factor $\tan \delta$ caused by non-synchronized sampling and non-integral period truncation. The limitations of Fast Fourier transform (FFT) application in measurements are due to spectral leakage and the picket-fence effect. Spectral leakage is typically reduced by selection of the proper window [10]. The picket-fence effect errors are compressed by interpolated FFT. The FFT interpolation formula for the rectangular window was introduced in [11]. It was then extended for a Hanning window in [12].It is also known that windows with a narrow main lobe have better noise immunity [13]. In this paper we have used some different window functions for evaluation and applied them on 3 different locations in satellite images.

\section{SubPIXEL REgISTRATION By PHASE CORRELATION METHOD}

To obtain the phase correlation function. Let the image $\mathrm{I}_{2}$ be a shifted version of the image $\mathrm{I}_{1}$ by $(x 0, y 0)$, then

$$
\mathrm{I}_{2}(\mathrm{x}, \mathrm{y})=\mathrm{I}_{1}(\mathrm{x}-\mathrm{x} 0, \mathrm{y}-\mathrm{y} 0)
$$

After taking the Fourier Transform (FT) of both images, we have the following relationship due to the shift property of the FT 


$$
\mathrm{I}_{2}(u, v)=\mathrm{I}_{1}(u, v) e^{-j(u x 0+v y 0)}
$$

Therefore, a shift in the spatial domain will produce a phase difference in the frequency domain. The normalized cross power spectrums finally denudes

$$
\frac{I_{2}(u, v) I_{1}(u, v)}{\left|I_{2}(u, v) I_{1}(u, v)\right|}=e^{-j(u x 0+v y o)}
$$

The (PC) function is finally obtained by taking the Inverse Fourier Transform (IFT) of the cross-power spectrum, which gives a $\delta(\mathrm{x} 0, \mathrm{y} 0)$ as result: a Dirac function centered on the position $(\mathrm{x} 0, \mathrm{y} 0)$.

None the less, appointed out in [5], when dealing with discrete images and using the Fast Fourier Transform (FFT) to generate the PC, the Dirac is turned into a Dirichlet kernel, whose maximum peak is found at the closest integer displacement, so finding the PC peak is equivalent to finding the translation at a pixel resolution. In order to obtain sub pixel resolution and keep using the same technique offending the peak position of the PC function, interpolation by zero padding the cross-power spectrum is suggested in [4], but accuracy is limited by the interpolation factor used which is also limited by the size of the IFFT that can be computed. Lately, this approach has been improved with a more efficient implementation proposed in [14]. Using a different approach, in[5] an extension of the original PC method is presented where using not only the PC information of the main peak, but also its surrounding pixels, leads to an estimation of the amount of sub pixel displacement as well. Let $\mathrm{C}(0,0)$ be the main peak, and $\mathrm{C}(1,0)$ and $\mathrm{C}(0,1)$ be the neighbors with the largest value in both horizontal and vertical direction respectively. The sub pixel displacement is then calculated as:

$$
\Delta x=\frac{c(1,0) * x p+c(0,0) * x}{c(1,0)+c(0,0)}-1 \quad \Delta y=\frac{c(0,1) * y p+c(0,0) * y}{c(0,1)+c(0,0)}-1
$$

Where $\mathrm{x}_{\mathrm{p}}$ positive $\mathrm{X}$ direction and $\mathrm{y}_{\mathrm{p}}$ positive $\mathrm{Y}$ direction.

\section{WINDOWS FUNCTION \& HIGH PASS FILTER BASED ON PHASE CORRELATION}

\section{A. Window Function}

In signal processing, a window function (also known as an apodization function or tapering function [15]) is a mathematical function that is zero-valued outside of some chosen interval. For instance, a function that is constant inside the interval and zero elsewhere is called a rectangular window, which describes the shape of its graphical representation. When another function or a signal (data) is multiplied by a window function, the product is also zero-valued outside the interval: all that is left is the part where they overlap; the "view through the window". Applications of window functions include spectral analysis, filter design, and beam forming. The following table describes the window functions [15].
TABLE I. WINDOWS FUNCTIONS.

\begin{tabular}{|c|c|}
\hline Window & Functions \\
\hline Triangle & $w[n]=\frac{2}{N+1} \cdot\left(\frac{N+1}{2}-\left|n-\frac{N-1}{2}\right|\right)$ \\
\hline Hanning & $w[n]=0.5\left(1-\cos \left[\frac{2 \pi n}{N}\right]\right)$ \\
\hline Hamming & $w[n]=0.5+(1-0.5) \cos \left[\frac{2 \pi n}{N}\right]$ \\
\hline Kaiser & $w[n]=\frac{I 0\left(3 \pi \sqrt{1-\left(\frac{2 n}{N-1}\right)^{2}}\right)}{I 0(3 \pi)}$ \\
\hline Chebwin & $w[n]=\frac{\cos \left(N * \cos { }^{-1}\left[\alpha * \cos \left(\frac{\pi m}{n}\right)\right]\right)}{\cosh \left[N * \cosh { }^{-1}(\alpha)\right]}$ \\
\hline Blackman & $w[n]=0.42+0.5 \cos \left[\frac{2 \pi n}{N}\right]+0.08 \cos \left[\frac{4 \pi n}{N}\right]$ \\
\hline Boxcar & $w[n]=R E C T\left[\frac{n}{0.97 N}\right]$ \\
\hline Bartlett & $w[n]=\frac{2}{N-1} \cdot\left(\frac{N-1}{2}-\left|n-\frac{N-1}{2}\right|\right)$ \\
\hline
\end{tabular}

\section{B. High Pass Filter}

A high pass filter (HPF) could be analog or digital filter. Analog filter should be electronic circuits to filter image before recording, on the other hand digital filter will deal with the image after being recorded, so here we are using digital filter. Filter could be Finite impulse response (FIR) or Infinite impulse response (IIR) $[16,17]$. The main difference is that FIR would have a linear phase but IIR will give non linear phase. In our method we need to make calculations on phase, so we have more concern in FIR to get better result and fast processing. For instance, a HPF will be used to reduce low frequency parts details in the image as desert is considered as the low frequency component in the data of the image.

\section{MATERIALS AND METHOD}

\section{A. Data Sets}

The French satellite SPOT5 was launched in 2002. It has a resolution of $5 \mathrm{~m}$ for the panchromatic band (HRG instrument) with an unchanged swath of $60 \mathrm{~km}$ [18]. Extracting the $2.5 \mathrm{~m}$ from $5 \mathrm{~m}$ images requires a special technique. We will use tow scenes; their characteristics are shown in Table 2.

TABLE II. IMAGE CHARACTERISTICS.

\begin{tabular}{|c|c|c|}
\hline Images & Image_1 & Images_2 \\
\hline $\mathrm{K}$ & 113 & 113 \\
\hline $\mathrm{J}$ & 289 & 289 \\
\hline Description & Panchromatic & Panchromatic \\
\hline Wavelength $(\mu \mathrm{m})$ & $0.48-0.71$ & $0.48-0.71$ \\
\hline Spectral mode & $\mathrm{A}$ & $\mathrm{B}$ \\
\hline Processing level & $1 \mathrm{~A}$ & $1 \mathrm{~A}$ \\
\hline Acquisition date & $16 / 06 / 2008$ & $16 / 06 / 2008$ \\
\hline
\end{tabular}




\section{B. Operation Steps}

Steps for phase correlation using window function \& high pass filters are as follows:

- $\quad$ Read image \& apply windows and high pass filter.

- Get 2D FFT for both images.

- Get phase correlation surface, defines as $\mathrm{C}_{\mathrm{t}, \mathrm{t}+1}(\mathrm{k}, \mathrm{l})=\mathrm{F}^{-1}\left(\frac{\mathrm{F}_{\mathrm{t}}^{*} \mathrm{~F}_{\mathrm{t}+1}}{\left|\mathrm{~F}_{\mathrm{t}}^{*} \mathrm{~F}_{\mathrm{t}+1}\right|}\right)$ where $\mathrm{Ft}$ and $\mathrm{Ft}+1$ are respectively the two-dimensional Fourier transforms of $\mathrm{F}_{\mathrm{t}}$ and $\mathrm{F}_{\mathrm{t}+1}, \mathrm{~F}^{-1}$ denotes the inverse Fourier transform and* denotes complex conjugate.

- Get maximum real point in 2D surface.

- $\quad$ Get position of this point $\mathrm{x}, \mathrm{y}$.

$$
\left(K_{m}, l_{m}\right)=\operatorname{argmax} \operatorname{Re}\left\{C_{t, t+1}(k, l)\right\}
$$

Where $\left(\mathrm{k}_{\mathrm{m}}, \mathrm{l}_{\mathrm{m}}\right)$ co-ordinates of the maximum value of array $C_{t, t+1}(k, l)$

- Calculate sub pixel difference $\Delta \mathrm{x}$ and $\Delta \mathrm{y}$ from eq. 4 .

The algorithm starts from the already shifted image. The roughly coregistered images (to the same reference system spot5) are correlated using a small sliding window. We apply a different window function on both images to reduce noise edge effects. We calculate the Fast Fourier transform FFT of both images. Then, Calculate the cross-power spectrum by taking the complex conjugate of the result. By multiplying the Fourier transforms together, element wise, we obtained phase correlation surface by applying the inverse Fourier transform. Finally we determined the location of the maximum peak from real value of surface $(x, y)$. The entire processing chain is summarized in Figure.1.

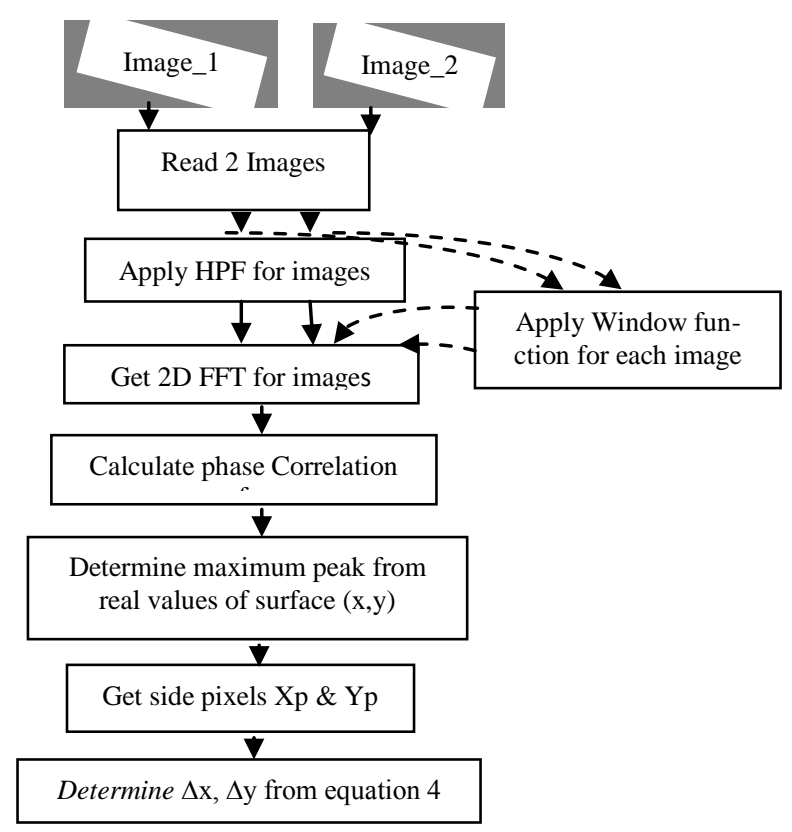

Figure .1 Algorithm workflow diagram of the proposed phase correlation based window function method and high pass filter

\section{DATA ANALYSIS}

We applied the previous algorithm In order to simulate the sub pixel displacements using a couple of panchromatic images $(2000 \times 2000$ pixels). The application was conducted using different windows, on shifted images as shown in Figure.2. These two images have"-0.5, 0.5 pixel" shift between them. There are many phenomena in the images that will affect the shift results as shown in Table.3.
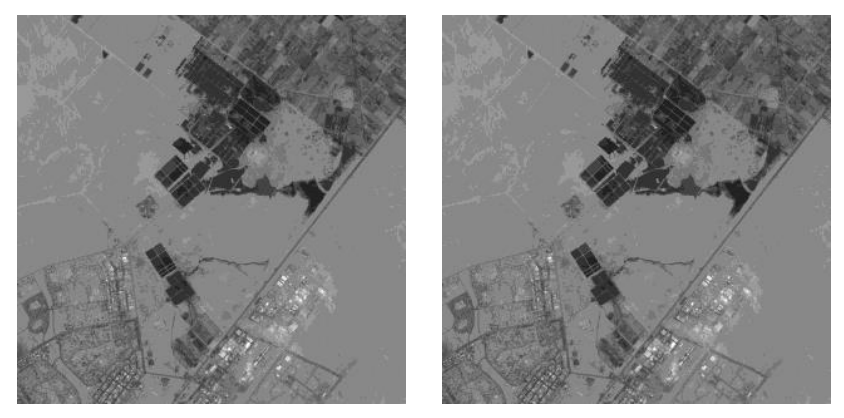

Figure.2. Two sample images shifted by"-0.5, 0.5" pixel.

TABLE III. OUTPUT WIDOWS SHIFT RESULTS OF THE SOURCE IMAGE.

\begin{tabular}{|l|c|c|}
\hline \multirow{2}{*}{ windows } & \multicolumn{2}{|c|}{ Source image } \\
\cline { 2 - 3 } & $\Delta \mathbf{x}$ & $\Delta \mathbf{y}$ \\
\hline No Window & -0.7450 & 0.7303 \\
\hline Triangle & -0.7784 & 0.7617 \\
\hline Hanning & -0.7874 & 0.7621 \\
\hline Hamming & -0.7819 & 0.7603 \\
\hline Kaiser & -0.8028 & 0.7771 \\
\hline Chebwin & -0.8056 & 0.7798 \\
\hline Blackman & -0.7986 & 0.7731 \\
\hline Boxcar & -0.7450 & 0.7303 \\
\hline Bartlett & -0.7784 & 0.7617 \\
\hline
\end{tabular}

VI. EVALUATION OF DIFFERENT AREA LOCATIONS

This section presents the evaluation of some windows functions on three different locations namely; (urban area, vegetation area and desert area).

\section{A. Urban area}

This area was chosen from the above data of Table. 2. The choice of this region was done because of the large number of urban areas, which have a lot of edges that makes the process faster in measuring the displacement between the two images. Figure. 3 shows the selected area $(500 \times 500)$ pixels, with different buildings and Figure. 4 depicts the urban pixel values with pixel location.

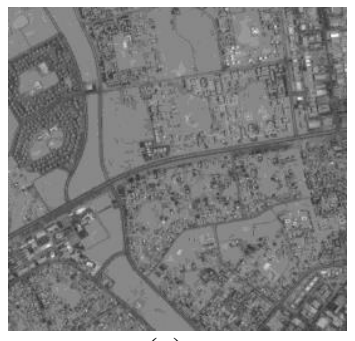

(a)

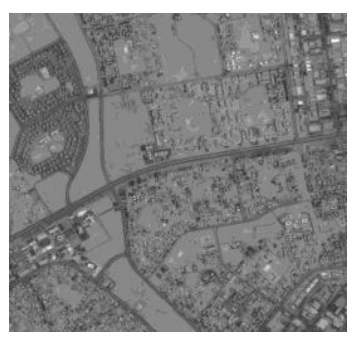

(b)
Figure.3.Two urban areas presenting roads, buildings and sharp edges. 


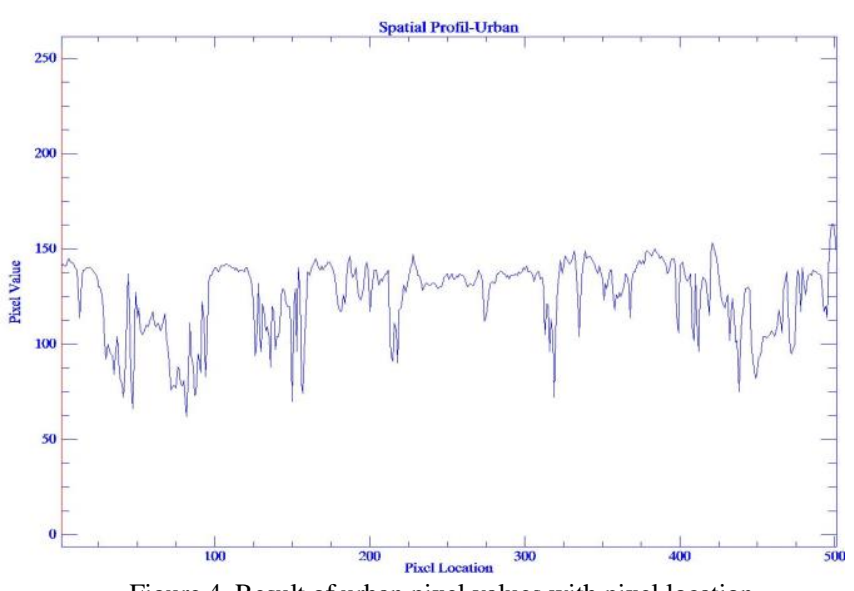

Figure.4. Result of urban pixel values with pixel location.

\section{B. Vegetation area}

This area was chosen from the above data of Table. 2. The Choice of this region was done because of the large number of Agricultural fields, in which the edges appear between the different parts in the fields, but not in abundance, such as buildings. Also the image size of these areas is $(500 \times 500)$ pixel.

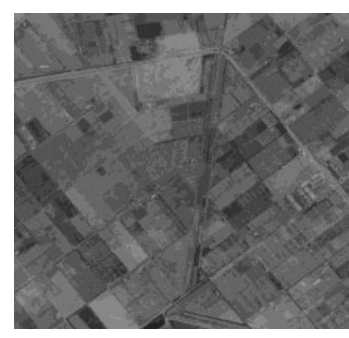

(a)

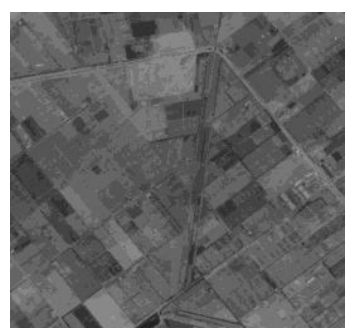

(b)
Figure.5. Two vegetation areas presenting fewer edges than urban.

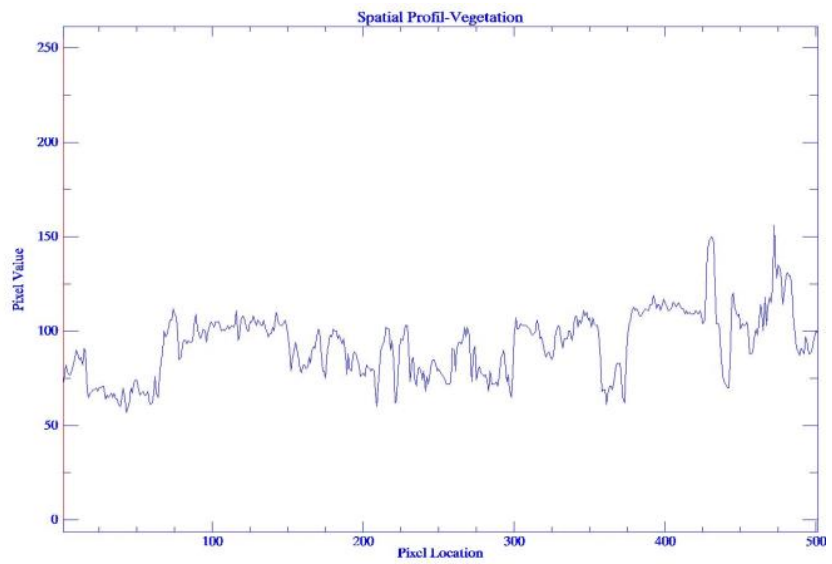

Figure.6. Result of vegetation pixel values with pixel location.

Figure.5 shows the vegetations area and Figure.6 depicts the vegetation pixel values with pixel location.

\section{Desert area}

This area was also chosen from the above data of Table 2. This desert region does not have edges that help successful completion of the measurements process.
Also the image size of this area is $(500 \times 500)$ pixel. Figure.7 shows the smoothed desert area and Figure. 8 depicts the desert pixel values with pixel location.

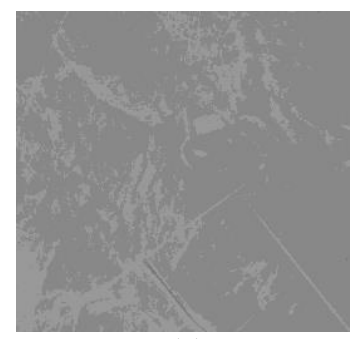

(a)

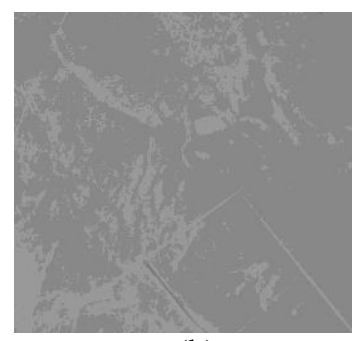

(b)
Figure.7. Two desert areas presenting no edges.

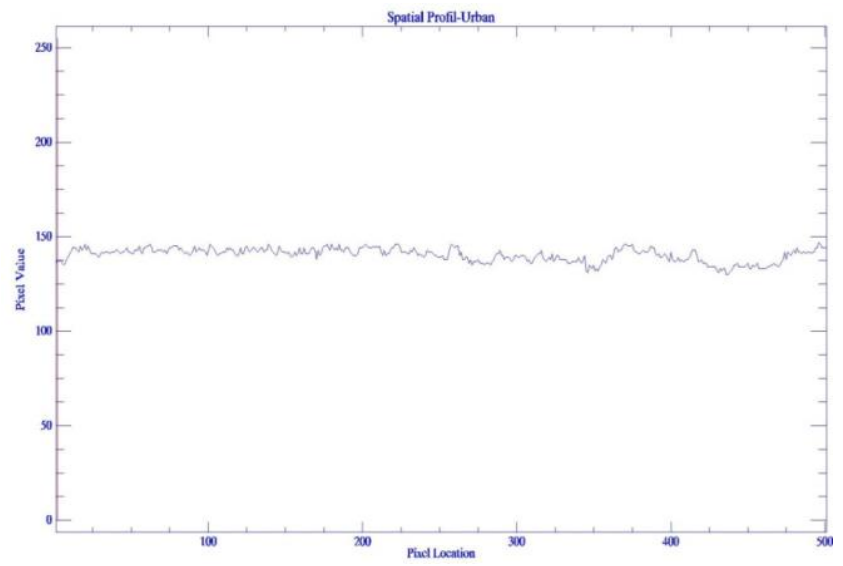

Figure.8. Result of desert pixel values with pixel location.

\section{RESULTS AND DISCUSSION}

\section{A. Urban area}

We applied the algorithm discussed in section 4 on the subset images of the buildings and roads. It has been found after using 8 different windows that; all windows are giving variable and different results from each other, but closer to the (actual shift) as shown in Table. 4, thanks to the large amount of edges produced by buildings.

\section{B. Vegetation area}

Also in this part the algorithm was applied to the fields of agriculture using the 8 different windows yielding poor result, as shown in Table.5. These results are due to the lack of edges that are on the expense of displacement calculation accuracy between the two images. However, as shown in the table without the use of windows, it gives a bad result, as well as using the Box windows.

\section{Desert area}

In case of the desert, we used the 8 different windows on areas where there are no edges which do not lead to satisfactory results and complete the measurement process successfully. Figure. 8 shows pixel values and there is no change that results from the edges. Therefore, results shown in Table. 6 are very bad for all the windows. 


\section{High pass filter (HPF)}

Finally we applied HPF (FIR filter) to overcome the problem of the desert, which has very bad results. Table.7 shows the results of using the filter on the different locations. It is clear that it gives a very comparable result.

TABLE IV. TRESULTS OF THE DIFFERENT WINDOWS IN URBAN AREA, $\triangle \mathrm{X}$ AND $\triangle Y$ ARE VALUES REPRESENTING THE SUB PIXEL SHIFT CALCULATED FROM THE DISCUSSED METHOD.

\begin{tabular}{|c|c|c|}
\hline \multirow{2}{*}{ Windows } & \multicolumn{2}{|c|}{ Urban } \\
\cline { 2 - 3 } & $\Delta \mathrm{x}$ & $\Delta \mathrm{y}$ \\
\hline No window & -0.5255 & 0.5057 \\
\hline Triangle & -0.5408 & 0.5501 \\
\hline Hanning & -0.5488 & 0.5573 \\
\hline Hamming & -0.5444 & 0.5507 \\
\hline Kaiser & -0.5589 & 0.5661 \\
\hline Chebwin & -0.5608 & 0.5674 \\
\hline Blackman & -0.5569 & 0.5643 \\
\hline Boxcar & -0.5255 & 0.5057 \\
\hline Bartlett & -0.5409 & 0.5503 \\
\hline
\end{tabular}

TABLE V. RESULT OF THE DIFFERENT WINDOWS IN THE VEGETATION AREA.

\begin{tabular}{|c|c|c|}
\hline \multirow{2}{*}{ windows } & \multicolumn{2}{|c|}{ Vegetation } \\
\cline { 2 - 3 } & $\Delta \mathrm{x}$ & $\Delta \mathrm{y}$ \\
\hline No window & -0.2587 & 0.4673 \\
\hline Triangle & -0.6861 & 0.7974 \\
\hline Hanning & -0.6910 & 0.7954 \\
\hline Hamming & -0.6878 & 0.7979 \\
\hline Kaiser & -0.6879 & 0.7908 \\
\hline Chebwin & -0.6864 & 0.7881 \\
\hline Blackman & -0.6903 & 0.7920 \\
\hline Boxcar & -0.2587 & 0.4673 \\
\hline Bartlett & -0.6863 & 0.7971 \\
\hline
\end{tabular}

TABLE VI. RESULT OF THE DIFFERENT WINDOWS IN THE DESERT AREA.

\begin{tabular}{|r|c|c|}
\hline \multirow{2}{*}{ windows } & \multicolumn{2}{|c|}{ Desert } \\
\cline { 2 - 3 } & $\Delta \mathrm{x}$ & $\Delta \mathrm{y}$ \\
\hline No window & 0.0518 & 0.4511 \\
\hline Triangle & -1.0692 & 1.0961 \\
\hline Hanning & -1.0804 & 1.0996 \\
\hline Hamming & -1.0729 & 1.0988 \\
\hline Kaiser & -1.0890 & 1.0973 \\
\hline Chebwin & -1.0885 & 1.0984 \\
\hline Blackman & -1.0880 & 1.0988 \\
\hline Boxcar & 0.0518 & 0.4511 \\
\hline Bartlett & -1.0695 & 1.0959 \\
\hline
\end{tabular}

TABLE VII. RESULTS OF THE DIFFERENT LOCATION USING HPF.

\begin{tabular}{|c|c|c|c|c|}
\hline$\Delta$ & $\begin{array}{c}\text { Source } \\
\text { image }\end{array}$ & $\begin{array}{c}\text { Urban } \\
\text { area }\end{array}$ & $\begin{array}{c}\text { Vegetation } \\
\text { area }\end{array}$ & Desert area \\
\hline$\Delta \mathrm{x}$ & -0.5014 & -0.5001 & -0.5093 & -0.4053 \\
\hline$\Delta \mathrm{y}$ & 0.5140 & 0.5014 & 0.5137 & 0.5013 \\
\hline
\end{tabular}

\section{CONCLUSION}

In this paper we present a algorithm for obtaining highaccuracy sub-pixel shit estimation using phase correlation. It appeared that having a large number of pixels gives a good representation of Fourier and using windows is not giving a better correlation solution in low frequency component (Desert). Results of applying the window functions did not succeed in enhancing the calculation of shift in images, but the (HPF) did make enhancement on source image by removing low frequency components hence helping us to calculate peak phase shift.

\section{REFERENCES}

[1] B. Zitova and J. Flusser, "Image registration methods: a survey," Image and Vision Computing, vol.21, no.11, pp.977-1000, October 2003.

[2] C. D. Kuglin and D. C. Hines, "The phase correlation image alignment method," in Proc. Int. Conference on Cybernetics and Society, pp. 163165, 1975.

[3] B. Reddy and B. Chatterji, "An fft-based technique for translation, rotation, and scale-invariant image registration," IEEE Trans. on Image Processing, vol. 5, no. 8, pp. 1266-1271, 1996.

[4] B. Marcel, M. Briot, and R. Murrieta, "Calcul de translation et rotation par la transformation de fourier," Traitement du Signal, vol. 14, no. 2, pp. 135-149, 1997.

[5] H. Foroosh, J. Zerubia, and M. Berthod, "Extension of phase correlation to subpixel registration," IEEE Trans. on Image Processing, vol. 11, no. 3, pp. 188-200, 2002.

[6] K. Takita, T. Aoki, Y. Sasaki, T. Higuchi, and K. Kobayashi, "Highaccuracy subpixel image registration based on phase-only correlation," IEICE Trans. Fund., vol. E86-A, no. 8, pp. 1925-1934, 2003.

[7] V. Argyriou and T. Vlachos, "Performance study of gradient correlation for sub-pixel motion estimation in the frequency domain," IEE Proc. Vision, Image, and Signal Processing, vol. 152, no. 1, pp. 107-114, 2005.

[8] V. Argyriou and T. Vlachos, "On the estimation of subpixel motion using phase correlation," Journal of Electronic Imaging, vol. 16, no. 033018, 2007.

[9] Y.H. Ha, J.A. Pearce," A new window and comparison to standard windows," IEEE Trans. Acoust., Speech Signal Process., 37, vol no.2, pp. 298-301,1989.

[10] F. J. Harris, "On the use of windows for harmonic analysis with the discrete Fourier transform," Proc. IEEE, vol. 66, no. 1, pp. 51-83, Jan. 1978.

[11] V. H. Jain, W. L. Collins, and D. C. Davis, "High accuracy analog measurement via interpolated FFT," IEEE Trans. Instrum. Meas., vol. IM-28, no. 2, pp. 113-122, Jun. 1979.

[12] T. Grandke, "Interpolation algorithms for discrete Fourier transform of weighted signals," IEEE Trans. Instrum. Meas., vol. IM-32, no. 2, pp. 350-355, Jun. 1983.

[13] K. duda " DFT Interpolation Algorithm for Kaiser-Bessel and DolphChebyshev Windows" IEEE Transaction on Instrumentation and Measurement, vol. 60, no. 3, March 2011.

[14] M. Guizar-Sicairos, S. T. Thurman, and J. R. Fienup, "Efficient subpixel image registration algorithms," Optics Letters, vol. 33, no. 2 , pp. $156-158,2008$.

[15] L. M. Surhon, M. T. Timpledon, S. F. Marseken,"window Function"ISBN 613030014X, 9786130300142, VDM Verlag, 2010, p.124.

[16] A. E. Cetin, O.N. Gerek, Y. Yardimci, "Equiripple FIR filter design by the FFT algorithm," IEEE Signal Processing Magazine, pp. 60-64, March 1997.

[17] Ranjit Singh, Sandeep K. Arya," Genetic Algorithm for the Design of Optimal IIR Digital Filters," Journal of Signal and Information Processing, pp. 286-292, August 2012.

[18] C. Fratter, M. Moulin, H. Ruiz, P. Charvet, D. Zobler, "The SPOT5 mission", 52nd International Astronautical Congress, Toulouse, France, 1-5 Oct 2001. 